Kragujevac Journal of Mathematics

Volume 39(2) (2015), Pages 133-139.

\title{
AN UPPER BOUND ON THE DOUBLE DOMINATION NUMBER OF TREES
}

\author{
J. AMJADI ${ }^{1}$
}

\begin{abstract}
In a graph $G$, a vertex dominates itself and its neighbors. A set $S$ of vertices in a graph $G$ is a double dominating set if $S$ dominates every vertex of $G$ at least twice. The double domination number $\gamma_{\times 2}(G)$ is the minimum cardinality of a double dominating set in $G$. The annihilation number $a(G)$ is the largest integer $k$ such that the sum of the first $k$ terms of the non-decreasing degree sequence of $G$ is at most the number of edges in $G$. In this paper, we show that for any tree $T$ of order $n \geq 2$, different from $P_{4}, \gamma_{\times 2}(T) \leq \frac{3 a(T)+1}{2}$.
\end{abstract}

\section{INTRODUCTION}

In this paper, $G$ is a simple graph with vertex set $V=V(G)$ and edge set $E=E(G)$. The order $|V|$ of $G$ is denoted by $n=n(G)$. For every vertex $v \in V(G)$, the open neighborhood of $v$ is the set $N_{G}(v)=N(v)=\{u \in V(G) \mid u v \in E(G)\}$ and the closed neighborhood of $v$ is the set $N_{G}[v]=N[v]=N(v) \cup\{v\}$. The degree of a vertex $v \in V$ is $\operatorname{deg}_{G}(v)=\operatorname{deg}(v)=|N(v)|$. The minimum degree of a graph $G$ is denoted by $\delta=\delta(G)$. A leaf of a tree $T$ is a vertex of degree 1 , a support vertex is a vertex adjacent to a leaf and a strong support vertex is a vertex adjacent to at least two leaves. A strong support vertex is said to be end-stem if all its neighbors except one of them are leaves. We write $P_{n}$ for a path of order $n$. For a subset $S \subseteq V(G)$, we let $\sum(S, G)=\sum_{v \in S} \operatorname{deg}_{G}(v)$. For notation and graph theory terminology, we in general follow [10].

The concept of domination in graphs, with its many variations, is now well studied in graph theory and the literature on this subject has been surveyed and detailed in the two books by Haynes, Hedetniemi, and Slater [10, 11]. A dominating set of a

Key words and phrases. domination number, double dominating set, double domination number, annihilation number, tree.

2010 Mathematics Subject Classification. 05C69.

Received: 23 April, 2015.

Accepted: 18 July, 2015. 
graph $G$ is a set $S$ of vertices of $G$ such that every vertex in $V(G)-S$ is adjacent to at least one vertex in $S$. The domination number of $G$, denoted by $\gamma(G)$, is the minimum cardinality of a dominating set of $G$.

Harary and Haynes [9] defined a generalization of domination as follows: a subset $S$ of $V$ is a $k$-tuple dominating set of $G$ if for every vertex $v \in V,|N[v] \cap S| \geq k$. The $k$ tuple domination number $\gamma_{\times k}(G)$ is the minimum cardinality of a $k$-tuple dominating set of $G$. Obviously, $\gamma(G)=\gamma_{\times 1}(G) \leq \gamma_{\times k}(G)$. For a graph to have a $k$-tuple dominating set, its minimum degree must be at least $k-1$. Hence for trees, we have $k \leq 2$. A 2-tuple dominating set is called a double dominating set (DDS). A $\gamma_{\times 2}(G)$ set is a DDS of cardinality $\gamma_{\times 2}(G)$. The redundancy involved in $k$-tuple domination makes it useful in many applications.

Let $d_{1}, d_{2}, \ldots, d_{n}$ be the degree sequence of a graph $G$ arranged in non-decreasing order, and so $d_{1} \leq d_{2} \leq \cdots \leq d_{n}$. The annihilation number of $G$, denoted $a(G)$, is the largest integer $k$ such that the sum of the first $k$ terms of the degree sequence is at most half the sum of the degrees in the sequence. Equivalently, the annihilation number is the largest integer $k$ satisfying the condition that $\sum_{i=1}^{k} d_{i} \leq \sum_{i=k+1}^{n} d_{i}$. It is immediate from the definition that if $G$ has $m$ edges and annihilation number $k$, then $\sum_{i=1}^{k} d_{i} \leq m$. The annihilation number was introduced by Pepper in [15] and has been studied by several authors $[2,6,7,8,13,14,16]$. As an immediate consequence of the definition of the annihilation number, Larson and Pepper [14] observed that for any graph $G$ of order $n, a(G) \geq\left\lfloor\frac{n}{2}\right\rfloor$.

In [15] and [16], Pepper proved that the annihilation number is an upper bound on the independence number of a graph and in [14] the case for equality of the upper bound was characterized by Larson and Pepper.

The relation between annihilation number and some graph parameters have been studied by several authors. For instance, DeLaViña et al. presented an upper bound on 2-domination number in terms of annihilation number for some classes of graphs [6], Dehgardi et al. investigated the relation between some domination parameters and the annihilation number of trees $[3,4,5]$, Desormeaux et al. proved that for any tree $T, a(T)+1$ is an upper bound on the total domination number [8].

If $G$ is a connected graph, different from $C_{5}$, of order $n$ with minimum degree at least two, then it is known [12] that $\gamma_{\times 2}(G) \leq \frac{3 n}{4}$. Hence, if $G \neq C_{5}$ is a connected graph of order $n$ with minimum degree at least 2 , then $\gamma_{\times 2}(G) \leq \frac{3 a(T)}{2}$ because $a(G) \geq\left\lfloor\frac{n}{2}\right\rfloor$. On the other hand, we have $\gamma_{\times 2}\left(C_{5}\right)=4=\frac{3 a\left(C_{5}\right)+2}{2}$.

In this paper we continue the study of double domination in trees and we prove that for any tree $T$ of order at least two $\gamma_{\times 2}(T) \leq \frac{3 a(T)+2}{2}$ and the equality holds if and only if $T=P_{4}$.

The value of $\gamma_{\times 2}\left(P_{n}\right)$ for a path $P_{n}$ is established in [1].

Proposition 1.1. $\gamma_{\times 2}\left(P_{n}\right)=2\left\lceil\frac{n}{3}\right\rceil+1$ if $n \equiv 0(\bmod 3)$ and $\gamma_{\times 2}\left(P_{n}\right)=2\left\lceil\frac{n}{3}\right\rceil$ otherwise. 
The annihilation number is easy to compute for paths and we have the following observation.

Observation 1.1. For $n \geq 2, a\left(P_{n}\right)=\left\lceil\frac{n}{2}\right\rceil$.

The next result is an immediate consequence of Proposition 1.1 and Observation 1.1.

Proposition 1.2. For $n \geq 3, \gamma_{\times 2}\left(P_{n}\right) \leq \frac{3 a(T)+2}{2}$ with equality if and only if $n=4$.

The next result is immediate from definitions.

Observation 1.2. Every leaf and every support vertex of a graph $G$ is in every $\gamma_{\times 2}(G)$ set.

A subdivision of an edge $u v$ is obtained by replacing the edge $u v$ with a path $u w v$, where $w$ is a new vertex. The subdivision graph $S(G)$ is the graph obtained from $G$ by subdividing each edge of $G$. The subdivision star $S\left(K_{1, t}\right)$ for $t \geq 2$, is called a healthy spider $S_{t, t}$. A wounded spider $S_{t, q}$ is the graph formed by subdividing $q$ of the edges of a star $K_{1, t}$ for $t \geq 2$ where $q \leq t-1$. Note that stars are wounded spiders. A spider is a healthy or wounded spider.

Proposition 1.3. If $T$ is a spider different from $P_{4}$, then $\gamma_{\times 2}(T) \leq \frac{3 a(T)+1}{2}$ with equality if and only if $T \cong S_{3,1}$ or $S_{4,3}$.

Proof. If $T=S_{t, t}$ is a healthy spider for some $t \geq 2$, then obviously $\gamma_{\times 2}(T)=2 t$ and $a(T)=t+\left\lfloor\frac{t}{2}\right\rfloor$, and we have $\gamma_{\times 2}(T) \leq \frac{3 a(T)}{2}$.

Now let $T=S_{t, q}$ be a wounded spider obtained from $K_{1, t}(t \geq 2)$ by subdividing $0 \leq q \leq t-1$ edges. Then $\gamma_{\times 2}(T)=n(T)$ by Observation 1.2. If $q=0$, then $T$ is a star and clearly $a(T)=t$ implying that $\gamma_{\times 2}(T) \leq \frac{3 a(T)}{2}$. Assume $q>0$. Since $T \neq P_{4}$, we have $q \neq 1$ or $t \neq 2$. It is easy to see that $a(T)=t+\left\lfloor\frac{q}{2}\right\rfloor$ and so $\gamma_{\times 2}(T) \leq \frac{3 a(T)+1}{2}$ with equality if and only if $T \cong S_{3,1}$ or $S_{4,3}$. This completes the proof.

For $p, q \geq 1$, a double star $D S_{p, q}$ is a tree with exactly two vertices that are not leaves, with one adjacent to $p$ leaves and the other to $q$ leaves.

Proposition 1.4. For the double star $D S_{p, q}$, different from $P_{4}$,

$$
\gamma_{\times 2}\left(D S_{p, q}\right) \leq \frac{3 a(T)+1}{2}
$$

with equality if and only if $T=D S_{1,2}$.

Proof. We may assume without loss of generality that $p \leq q$. Since $T \neq P_{4}, q \geq 2$. By Observation 1.2, $\gamma_{\times 2}\left(D S_{p, q}\right)=p+q+2$. On the other hand, $a\left(D S_{p, q}\right)=p+q$. This implies that $\gamma_{\times 2}\left(D S_{p, q}\right) \leq \frac{3 a(T)+1}{2}$ with equality if and only if $p=1$ and $q=2$, that is, $T=D S_{1,2}$. 


\section{An UpPer BOUnd}

In this section we establish an upper bound on the double domination number of trees in terms of their annihilation number.

For a vertex $v$ in a rooted tree $T$, let $C(v)$ denote the set of children of $v, D(v)$ denote the set of descendants of $v$ and $D[v]=D(v) \cup\{v\}$, and the depth of $v$, $\operatorname{depth}(v)$, is the largest distance from $v$ to a vertex in $D(v)$. The maximal subtree at $v$ is the subtree of $T$ induced by $D[v]$, and is denoted by $T_{v}$. In the sequel, we denote by $T-T_{v}$, the tree obtained from a rooted tree $T$ by deleting all vertices of $D[v]$.

In the proof of next theorem, we will always consider trees $T^{\prime}$ formed from $T$ by removing a set of vertices. For such a tree $T^{\prime}$ of order $n^{\prime}$, let $d_{1}^{\prime}, d_{2}^{\prime}, \ldots, d_{n^{\prime}}^{\prime}$ be the non-decreasing degree sequence of $T^{\prime}$, and let $S^{\prime}$ be a set of vertices corresponding to the first $a\left(T^{\prime}\right)$ terms in the degree sequence of $T^{\prime}$. In fact, if $u_{1}, u_{2}, \ldots, u_{n^{\prime}}$ are the vertices of $T^{\prime}$ such that $\operatorname{deg}\left(u_{i}\right)=d_{i}^{\prime}$ for each $i$, then $S^{\prime}=\left\{u_{1}, u_{2}, \ldots, u_{a\left(T^{\prime}\right)}\right\}$. We denote the size of $T^{\prime}$ by $m^{\prime}$.

Theorem 2.1. For any nontrivial tree $T$, different from $P_{4}, \gamma_{\times 2}(T) \leq \frac{3 a(T)+1}{2}$.

Proof. The proof is by induction on $n$. The statement holds for all trees of order $n \leq 4$. For the induction hypothesis, let $n \geq 5$ and suppose that for every nontrivial tree $T$ of order less than $n$ the result is true. Let $T$ be a tree of order $n$. By Propositions $1.2,1.3$ and 1.4, we may assume $\operatorname{diam}(T) \geq 4$ and that $T$ is not a path. We proceed further with a series of claims that we may assume satisfied by the tree.

Claim 1. T has no end-steam.

Proof. Let $T$ have an end-stem $u$ and let $N(u)=\left\{v, u_{1}, u_{2}, \ldots, u_{k}\right\}$ where $v$ is not a leaf. Assume $T^{\prime}=T-\left\{u, u_{1}, \ldots, u_{k}\right\}$. If $T^{\prime}=P_{4}$, then clearly $\gamma_{\times 2}(T)=4+k$ and $a(T)=k+2$ implying that $\gamma_{\times 2}(T) \leq \frac{3 a(T)}{2}$. Let $T^{\prime} \neq P_{4}$. Then obviously $\gamma_{\times 2}(T) \leq \gamma_{\times 2}\left(T^{\prime}\right)+k+1$. If $v \notin S^{\prime}$, then $\sum\left(S^{\prime}, T\right)=\sum\left(S^{\prime}, T^{\prime}\right)$ and if $v \in S^{\prime}$, then $\sum\left(S^{\prime}, T\right)=\sum\left(S^{\prime}, T^{\prime}\right)+1$. Thus, $\sum\left(S^{\prime}, T\right)-1 \leq \sum\left(S^{\prime}, T^{\prime}\right) \leq m^{\prime}=m-k-1$, and hence $\sum\left(S^{\prime}, T\right) \leq m-k$. Let $S=S^{\prime} \cup\left\{u_{1}, \ldots, u_{k}\right\}$. Then $\sum(S, T)=\sum\left(S^{\prime}, T\right)+k \leq$ $m$ implying that $a(T) \geq a\left(T^{\prime}\right)+k$. By inductive hypothesis, we have

$\gamma_{\times 2}(T) \leq \gamma_{\times 2}\left(T^{\prime}\right)+k+1 \leq \frac{3 a\left(T^{\prime}\right)+1}{2}+k+1 \leq \frac{3(a(T)-k)+1}{2}+k+1 \leq \frac{3 a(T)+1}{2}$,

as desired.

Let $v_{1} v_{2} \ldots v_{D}$ be a diametral path in T. By Claim $1, \operatorname{deg}\left(v_{2}\right)=\operatorname{deg}\left(v_{D-1}\right)=2$ and all neighbors of $v_{3}$ with exception of $v_{4}$ (resp. all neighbors of $v_{D-2}$ with exception of $\left.v_{D-3}\right)$, are leaves or support vertices of degree 2 . If $\operatorname{diam}(T)=4$, then $T$ is a spider and the result follows by Proposition 1.3. Hence, we assume $\operatorname{diam}(T) \geq 5$. Root $T$ at $v_{D}$.

Claim 2. $\operatorname{deg}_{T}\left(v_{3}\right) \leq 3$. 
Proof. Assume that $\operatorname{deg}_{T}\left(v_{3}\right) \geq 4$. Let first $v_{3}$ be adjacent to a support vertex $w_{2} \notin\left\{v_{2}, v_{4}\right\}$. By Claim 1, we have $\operatorname{deg}_{T}\left(w_{2}\right)=2$. Suppose $w_{1}$ is the leaf adjacent to $w_{2}$ and let $T^{\prime}=T-\left\{v_{1}, v_{2}, w_{1}, w_{2}\right\}$. If $T^{\prime}=P_{4}$, then clearly $\gamma_{\times 2}(T) \leq 8$ and $a(T)=5$ implying that $\gamma_{\times 2}(T) \leq 8 \leq \frac{3 a(T)+1}{2}$. Let $T^{\prime} \neq P_{4}$. Then every double dominating set of $T^{\prime}$ can be extended to a double dominating set of $T$ by adding $v_{1}, w_{1}, v_{2}, w_{2}$ and hence $\gamma_{\times 2}(T) \leq \gamma_{\times 2}\left(T^{\prime}\right)+4$. Assume that $S=S^{\prime} \cup\left\{v_{1}, v_{2}, w_{1}\right\}$ when $v_{3} \notin S^{\prime}$ and $S=\left(S^{\prime}-\left\{v_{3}\right\}\right) \cup\left\{v_{1}, v_{2}, w_{1}, w_{2}\right\}$ if $v_{3} \in S^{\prime}$. Clearly $\sum(S, T) \leq m$ that implies $a(T) \geq|S|=\left|S^{\prime}\right|+3=a\left(T^{\prime}\right)+3$. It follows from the induction hypothesis that

$$
\gamma_{\times 2}(T) \leq \gamma_{\times 2}\left(T^{\prime}\right)+4 \leq \frac{3 a\left(T^{\prime}\right)+1}{2}+4 \leq \frac{3(a(T)-3)+1}{2}+4<\frac{3 a(T)+1}{2} .
$$

Now let all neighbors of $v_{3}$ with exception of $v_{2}, v_{4}$, are leaves. Suppose $w$ is a leaf adjacent to $v_{3}$ and let $T^{\prime}=T-\left\{v_{1}, v_{2}, w\right\}$. Since $\operatorname{daim}(T) \geq 5$, we have $T^{\prime} \neq P_{4}$. Clearly, every double dominating set of $T^{\prime}$ can be extended to a double domination set of $T$ by adding the vertices $v_{1}, v_{2}, w$ and hence $\gamma_{\times 2}(T) \leq \gamma_{\times 2}\left(T^{\prime}\right)+3$. Assume that $S=S^{\prime} \cup\left\{v_{1}, w\right\}$ when $v_{3} \notin S^{\prime}$ and $S=\left(S^{\prime}-\left\{v_{3}\right\}\right) \cup\left\{v_{1}, v_{2}, w\right\}$ if $v_{3} \in S^{\prime}$. Then $\sum(S, T) \leq m$ that implies $a(T) \geq|S|=\left|S^{\prime}\right|+2=a\left(T^{\prime}\right)+2$. As above, we have $\gamma_{\times 2}(T) \leq \frac{3 a(T)+1}{2}$.

Claim 3. $\operatorname{deg}_{T}\left(v_{3}\right)=2$.

Proof. Let $\operatorname{deg}\left(v_{3}\right)=3$. Suppose first that $v_{3}$ is adjacent to a support vertex $w_{2} \notin$ $\left\{v_{2}, v_{4}\right\}$. Let $w_{1}$ be the leaf adjacent to $w_{2}$ and $T^{\prime}=T-T_{v_{3}}$. If $T^{\prime}=P_{4}$, then clearly $\gamma_{\times 2}(T)=8$ and $a(T)=5$ implying that $\gamma_{\times 2}(T)=\frac{3 a(T)+1}{2}$. Let $T^{\prime} \neq P_{4}$. Then every double dominating set of $T^{\prime}$ can be extended to a double dominating set of $T$ by adding $v_{1}, w_{1}, v_{2}, w_{2}$ and hence $\gamma_{\times 2}(T) \leq \gamma_{\times 2}\left(T^{\prime}\right)+4$. Assume that $S=S^{\prime} \cup\left\{v_{1}, v_{2}, w_{1}\right\}$. Then $\sum(S, T) \leq m$ and so $a(T) \geq|S|=\left|S^{\prime}\right|+3=a\left(T^{\prime}\right)+3$. Now the result follows by the induction hypothesis.

Now let $v_{3}$ be adjacent to a leaf $w$ and let $T^{\prime}=T-\left\{v_{1}, v_{2}, w\right\}$. If $T^{\prime}=P_{4}$, then clearly $\gamma_{\times 2}(T)=6$ and $a(T)=5$ implying that $\gamma_{\times 2}(T)=6<\frac{3 a(T)+1}{2}$. Let $T^{\prime} \neq P_{4}$. Using an argument similar to that described in Claim 2, we obtain $\gamma_{\times 2}(T) \leq \frac{3 a(T)+1}{2}$ as desired.

Claim 4. There is no path $v_{4} z_{3} z_{2} z_{1}$ in $T$ such that $z_{3} \notin\left\{v_{3}, v_{5}\right\}$.

Proof. Assume there is a path $v_{4} z_{3} z_{2} z_{1}$ in $T$ where $z_{3} \notin\left\{v_{3}, v_{5}\right\}$. By Claims 1-3, we may assume $\operatorname{deg}_{T}\left(z_{2}\right)=\operatorname{deg}_{T}\left(z_{3}\right)=2$. Assume $T^{\prime}=T-\left\{v_{1}, v_{2}, v_{3}, z_{1}, z_{2}\right\}$. If $T^{\prime}=P_{4}$, then clearly $\gamma_{\times 2}(T)=7$ and $a(T)=5$, implying that $\gamma_{\times 2}(T)<\frac{3 a(T)+1}{2}$. Let $T^{\prime} \neq P_{4}$. By Observation 1.2, $v_{4}$ is in every $\gamma_{\times 2}(T)$-set because $v_{4}$ is a support vertex. Hence, every $\gamma_{\times 2}\left(T^{\prime}\right)$-set can be extended to a double dominating set of $T$ by adding the vertices $v_{1}, v_{2}, z_{1}, z_{2}$ which implies that $\gamma_{\times 2}(T) \leq \gamma_{\times 2}\left(T^{\prime}\right)+4$. Assume $S=S^{\prime} \cup\left\{v_{1}, v_{2}, z_{1}\right\}$ when $v_{4} \notin S^{\prime}$ and $S=\left(S^{\prime}-\left\{v_{4}\right\}\right) \cup\left\{v_{1}, v_{2}, v_{3}, z_{1}\right\}$ when $v_{4} \in S^{\prime}$. Then $\sum(S, T) \leq m$ and hence $a(T) \geq a\left(T^{\prime}\right)+3$. Now the result follows by inductive hypothesis. 
Claim 5. $v_{4}$ is not a support vertex.

Proof. Assume $v_{4}$ is a support vertex. Let $T^{\prime}=T-\left\{v_{1}, v_{2}, v_{3}\right\}$. If $T^{\prime}=P_{4}$, then it is easy to verify that $\gamma_{\times 2}(T) \leq \frac{3 a(T)+1}{2}$. Let $T^{\prime} \neq P_{4}$. By Observation $1.2, v_{4}$ is in every $\gamma_{\times 2}\left(T^{\prime}\right)$-set and so every $\gamma_{\times 2}\left(T^{\prime}\right)$-set can be extended to a double dominating set of $T$ by adding the vertices $v_{1}, v_{2}$ implying that $\gamma_{\times 2}(T) \leq \gamma_{\times 2}\left(T^{\prime}\right)+2$. Assume $S=S^{\prime} \cup\left\{v_{1}, v_{2}\right\}$ when $v_{4} \notin S^{\prime}$ and $S=\left(S^{\prime}-\left\{v_{4}\right\}\right) \cup\left\{v_{1}, v_{2}, v_{3}\right\}$ when $v_{4} \in S^{\prime}$. Clearly $\sum(S, T) \leq m$ and hence $a(T) \geq a\left(T^{\prime}\right)+2$. Applying the induction hypothesis we obtain $\gamma_{\times 2}(T)<\frac{3 a(T)+1}{2}$.

Claim 6. $v_{4}$ is not adjacent to a support vertex other than $v_{5}$.

Proof. Let $v_{4}$ be adjacent to a support vertex, say $w$ and let $T^{\prime}=T-\left\{v_{1}, v_{2}\right\}$. Suppose $D$ is a $\gamma_{\times 2}\left(T^{\prime}\right)$-set. Then $v_{3}, v_{4}, w \in D$ and obviously $\left(D-\left\{v_{3}\right\}\right) \cup\left\{v_{1}, v_{2}\right\}$ is a DDS of $T$ and hence $\gamma_{\times 2}(T) \leq \gamma_{\times 2}\left(T^{\prime}\right)+1$. Assume $S=S^{\prime} \cup\left\{v_{1}\right\}$. Then $\sum(S, T) \leq m$ that implies $a(T) \geq a\left(T^{\prime}\right)+1$. By the induction hypothesis we have $\gamma_{\times 2}(T)<\frac{3 a(T)+1}{2}$.

By Claims 4, 5, and 6 we may assume $\operatorname{deg}_{T}\left(v_{4}\right)=2$. Similarly, by rooting $T$ at $v_{1}$, we may assume that $\operatorname{deg}\left(v_{D-1}\right)=\operatorname{deg}\left(v_{D-2}\right)=\operatorname{deg}\left(v_{D-3}\right)=2$.

We now return to the proof of theorem. If $\operatorname{diam}(T)=5,6$ or 7 then $T$ is a path of order 6,7 and 8, respectively, and the result is immediate by Proposition 1.2. Let $\operatorname{diam}(T) \geq 8$ and $T^{\prime}=T-\left\{v_{1}, v_{2}, v_{3}, v_{D}, v_{D-1}, v_{D-2}\right\}$. If $T^{\prime}=P_{4}$, then obviously $T$ is a path of order 10 and the result follows by Proposition 1.2. Assume $T^{\prime} \neq P_{4}$. Since $\operatorname{deg}_{T^{\prime}}\left(v_{4}\right)=\operatorname{deg}_{T^{\prime}}\left(v_{D-3}\right)=1$, every $\gamma_{\times 2}\left(T^{\prime}\right)$-set contains $v_{4}, v_{D-3}$ and can be extended to a dominating set of $T$ by adding $v_{1}, v_{2}, v_{D}, v_{D-1}$ which implies that $\gamma_{\times 2}(T) \leq \gamma_{\times 2}\left(T^{\prime}\right)+4$. Suppose $S=S^{\prime} \cup\left\{v_{1}, v_{2}, v_{D}\right\}$. Then $\sum(S, T) \leq m$ and so $a(T) \geq a\left(T^{\prime}\right)+3$. Applying the induction hypothesis, we obtain $\gamma_{\times 2}(T) \leq$ $\gamma_{\times 2}\left(T^{\prime}\right)+4 \leq \frac{3 a\left(T^{\prime}\right)+1}{2}+4 \leq \frac{3(a(T)-3)+1}{2}+4<\frac{3 a(T)+1}{2}$. This completes the proof.

The coronal of two graphs $G_{1}$ and $G_{2}$, is the graph $G=G_{1} \circ G_{2}$ formed from one copy of $G_{1}$ and $\left|V\left(G_{1}\right)\right|$ copies of $G_{2}$ where the $i$ th vertex of $G_{1}$ is adjacent to every vertex in the $i$ th copy of $G_{2}$.

Assume that $P_{n}=v_{1} v_{2} \ldots v_{n}$ is a path on $n$ vertices and let $G_{n}=P_{n} \circ K_{1}$. Suppose $u_{i}$ is the leaf adjacent to $v_{i}$ for each $i$.

Theorem 2.2. For $k \geq 3$, there exists a tree $T$ with $\operatorname{daim}(T)=k$ and $\gamma_{\times 2}(T)=\frac{3 a(T)+1}{2}$.

Proof. Let $k \geq 3$ be an integer. If $k \equiv 2(\bmod 3)$, then let $T=G_{k-1}$. It is easy to see that $\gamma_{\times 2}(T)=2 k-2$ and $a(T)=\frac{4(k-2)}{3}+1$, and so $\gamma_{\times 2}(T)=\frac{3 a(T)+1}{2}$. If $k \equiv 0(\bmod 3)$, then let $T$ be the tree obtained from $G_{k-1}$ by adding a pendant edge at $v_{1}$. It is not hard to see that $\gamma_{\times 2}(T)=2 k-1$ and $a(T)=\frac{4 k}{3}-1$, and hence $\gamma_{\times 2}(T)=\frac{3 a(T)+1}{2}$. Finally, if $k \equiv 1(\bmod 3)$, then let $T$ be the tree obtained from $G_{k-1}$ by adding a pendant edge at $v_{1}$ and a pendant edge at $v_{k-1}$. It is easy to see that $\gamma_{\times 2}(T)=2 k$ and $a(T)=\frac{4(k-1)}{3}+1$, and hence $\gamma_{\times 2}(T)=\frac{3 a(T)+1}{2}$. This completes the proof. 
We conclude this paper with an open problem.

Conjecture 1. For any connected graph $G$ of order $n \geq 2$ with $\delta(G)=1$, $\gamma_{\times 2}(G) \leq \frac{3 a(G)+2}{2}$.

\section{REFERENCES}

[1] M. Blidia, M. Chellali, T. W. Haynes and M. A. Henning, Independent and double domination in trees, Util. Math. 70 (2006) 159-173.

[2] Y. Caro and R. Pepper, Degree sequence index strategy, Australas. J. Combin. 59(1) (2014) $1-23$.

[3] N. Dehgardi, S. M. Sheikholeslami and A. Khodkar, Bounding the rainbow domination number of a tree in terms of its annihilation number, Trans. Comb. 2 (2013), 21-32.

[4] N. Dehgardi, S. M. Sheikholeslami and A. Khodkar, Bounding the paired-domination number of a tree in terms of its annihilation number, Filomat 28 (2014), 523-529.

[5] N. Dehgardi, S. Norouzian and S. M. Sheikholeslami, Bounding the domination number of a tree in terms of its annihilation number, Trans. Comb. 2 (2013), 9-16.

[6] E. DeLaVina, C. E. Larson, R. Pepper and B. Waller, Graffiti.pc on the 2-domination number of a graph, Congr. Numer. 203 (2010), 15-32.

[7] E. DeLaVina, R. Pepper and B. Waller, Independence, radius, and Hamiltonian paths, MATCH Commun. Math. Comput. Chem. 58 (2007), 481-510.

[8] W. J. Desormeaux, T. W. Haynes and M. A. Henning, Relating the annihilation number and the total domination number of a tree, Discrete Appl. Math. 161 (2013), 349-354.

[9] F. Harary and T. W. Haynes, Double domination in graphs, Ars Combin. 55 (2000) 201-213.

[10] T. W. Haynes, S. T. Hedetniemi and P. J. Slater, Fundamentals of Domination in Graphs, Marcel Dekker, Inc., New York, 1998.

[11] T. W. Haynes, S. T. Hedetniemi, and P. J. Slater, Domination in Graphs: Advanced Topics, Marcel Dekker, Inc., New York, 1998.

[12] M. A. Henning, Graphs with large double domination numbers, Discuss. Math. Graph Theory 25 (2005), 13-28.

[13] L. Jennings, New Sufficient Condition for Hamiltonian Paths, Ph.D. Dissertation, Rice University, 2008.

[14] C. E. Larson and R. Pepper, Graphs with equal independence and annihilation numbers, Electron. J. Combin. 18 (2011), \#P180.

[15] R. Pepper, Binding Independence, Ph.D. Dissertation, University of Houston, 2004.

[16] R. Pepper, On the annihilation number of a graph, Recent Advances In Electrical Engineering: Proceedings of the 15th American Conference on Applied Mathematics, 2009, pp. 217-220.

[17] B. Reed, Paths, stars and the number three, Combin. Probab. Comput. 5 (1996), 277-295.

${ }^{1}$ Department of Mathematics,

Azarbaijan Shahid Madani University,

TABRIZ, I.R. IRAN

E-mail address: j-amjadi@azaruniv.edu 